\title{
Single Byte Differential Fault Analysis on the LED Lightweight Cipher in the Wireless Sensor Network
}

\author{
Wei $\mathbf{L i}$ \\ School of Computer Science and Technology, Donghua University \\ Shanghai, 201620, China \\ Shanghai Key Laboratory of Integrate Administration Technologies for Information Security \\ Shanghai, 200240, China
}

State Key Laboratory of Information Security, Institute of Information Engineering, Chinese Academy of Sciences

Beijing 100093, China

Da-wu Gu

Department of Computer Science and Engineering, Shanghai Jiao Tong University Shanghai, 200240, China

Xiao-ling Xia ${ }^{*}$, Chen Zhao

School of Computer Science and Technology, Donghua University

Shanghai, 201620, China

E-mail: sherlysha@dhu.edu.cn

Zhi-qiang Liu, Ya Liu

Department of Computer Science and Engineering, Shanghai Jiao Tong University

Shanghai, 200240, China

Qing-ju Wang

Department of Electrical Engineering ESAT/SCD-COSIC, Katholieke Universiteit Leuven

Heverlee, B-3001, Belgium

Received 15 December 2011

Accepted 15 June 2012

\begin{abstract}
The LED is a new lightweight cipher, which was published in CHES 2011. This cipher could be applied in the Wireless Sensor Network to provide security. On the basis of the single byte-oriented fault model, we propose a differential fault analysis on the LED cipher. The attack could recover its 64-bit secret key by introducing 4 faulty ciphertexts, and 128-bit secret key by introducing 8 faulty ciphertexts. The results in this study will be beneficial to the analysis of the same type of other iterated lightweight ciphers.
\end{abstract}

Keywords: Wireless Sensor Network; Lightweight Cipher; LED; Differential Fault Analysis.

\section{Introduction}

\footnotetext{
* Corresponding Author: Xiaoling Xia.
}

The LED is a new Substitution-Permutation Network (SPN) lightweight cipher published in CHES 2011 ${ }^{1}$. It has a good compact hardware implementation and 
software-friendly features, and thus could be applied in the sensor network, RIFD tag deployment and other application characterized by highly-constrained devices. The strength of the LED cipher against various classical cryptanalysis has been analyzed, including differential cryptanalysis, linear cryptanalysis, algebraic attack and so on ${ }^{1}$.

Other than classical cryptanalysis, differential fault analysis (DFA) is a new class of cryptanalysis on cryptographic devices. It was first proposed by E. Biham and A. Shamir on $\mathrm{DES}^{2}$ in 1997. The similar attacks have been applied to $\mathrm{AES}^{3-8}$, Triple-DES ${ }^{9}$, RC4 ${ }^{10}$, Camellia $^{11}$, ARIA ${ }^{12}$, SMS $^{13-14}{ }^{\text {, PRESENT }}{ }^{15}$ and so on. The DFA exploits easily accessible information like input-output behavior under malfunctions, amplifies and evaluates the leaked information with the help of mathematical methods. It is based on deriving information about the secret key by examining the differences between a cipher resulting from correct operation and a cipher of the same initial message resulting from faulty operation. This analysis is often much more powerful than classical cryptanalysis.

As for the SPN block ciphers, there are two types of DFA methods, which relate to the recovery of subkeys:

(i) The bit number of the fault is less than or equals to that of the input of an S-box. For example, the fault is one bit or byte, while the input of an S-box is one byte. In this case, one fault's diffusion affects at most one S-box in the inducing round, and the fault propagation path is simple. Thus, it is not difficult for the attackers to derive the relationship between the subkeys and the ciphertext difference. Many studies have been conducted on the security of these SPN block ciphers against the DFA ${ }^{3-8,12,15}$.

(ii) The bit number of the fault is greater than that of the input of an S-box. For example, the fault is a random byte, while the input of an S-box is 4 bits. Thus, the fault propagation paths may intersect each other. Thus it is not easy to deduce the relationship between the secret key and the ciphertext difference. For example, The LED is such a representative SPN block cipher which chooses 4 bits as a processing unit and its input of an S-box has 4 bits for the high efficiency in software and hardware. In the real circumstances, it is usual for the DFA attacker to choose the singlebyte oriented fault model to deduce the subkeys and the secret key for its easy implementation and general applications. However, few studies have been done on the single byte differential fault analysis on the LED cipher.

In this study, we propose a single byte differential fault analysis method to recover the secret key of LED. The method could induce one-byte errors into the encryption. Both the locations and the values of the errors are unknown. By retrieving the related values of subkeys, our method requires 4 and 8 ciphertexts to recover the 64-bit and 128-bit secret keys of LED, respectively.

The rest of this paper is organized as follows. Section 2 briefly introduces the LED cipher. The next section describes the basic assumption and basic idea of DFA. Then section 4 proposes our DFA analysis to recover the secret key. Section 5 summarizes the attacking complexity. Section 6 shows the experimental results of the DFA on LED. Finally section 7 concludes the paper.

\section{Description of the LED Cipher}

The LED is a 64-bit SPN block cipher with two primary instances taking 64-bit and 128-bit secret keys. It has $l$ rounds, which is 32 for LED-64 and 48 for LED-128 as Fig. 1 shows. The cipher is composed of encryption, decryption and the key schedule.

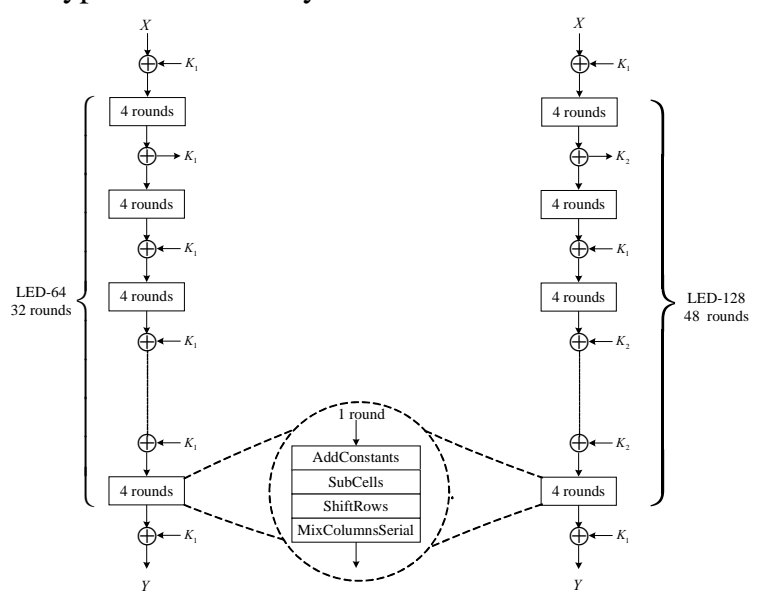

Fig. 1. The structure of LED-64 and LED-128.

\subsection{Encryption}

The LED could be pictured as a rectangular array of 4 bits, having four rows and four columns. Let $X$ be the plaintext and $Y$ be the ciphertext. Let $K_{1}$ and $K_{2}$ denote the subkeys from the secret key $K$. The intermediate result is denoted as $S T A T E \in\left(\{0,1\}^{4}\right)^{16}$. In every four rounds, the STATE is XORed with a 64-bit subkey, 
called AddRoundKey. The basic component of LED is a sequence of four identical rounds, and each round includes AddConstants, SubCells, ShiftRows and MixColumnsSerial in sequence:

(i) AddConstants is the transformation that processes the STATE with a constant.

(ii) SubCells is the transformation that processes a STATE with a nonlinear 4-bit substitution table (Sbox).

(iii) ShiftRows is the transformation that cyclically shifts the last three rows of the STATE by different offsets.

(iv) MixColumnsSerials is the transformation that takes all the columns of the STATE and mixes their data to produce new columns.

\subsection{Decryption}

The decryption is the same as that of encryption, including the subkeys with the same order.

\subsection{Key Schedule}

The secret key $K$ is the input of a key schedule to produce the subkeys for each round. In LED-64, the relationship between the secret key $K$ and the subkey $K_{1}$ is as follows:

$$
K=K_{1} .
$$

In LED-128, the relationship among the secret key $\mathrm{K}$, the subkeys $K_{1}$ and $K_{2}$ is as follows:

$$
K=K_{1} \| K_{2} \text {. }
$$

\section{Basic Idea of Our Attack}

\subsection{The Basic Assumption}

The DFA analysis exploits the difference between a normal and a faulty ciphertext stemming from encryptions of the same plaintext. Our proposed fault model includes the following two assumptions:

(i) The attacker has the capability to choose one plaintext to encrypt and obtain the corresponding right and faulty ciphertexts (Chosen Plaintext Attack, CPA).

(ii) The attacker could induce a single byte error to one transformation. However, the location of this byte in this round and the value of the error are both unknown.

\subsection{The Basic Idea}

The main procedure of this attack is as follows:

(i) The right ciphertext is obtained when a plaintext is encrypted with a secret key. (ii) We induce a random error in the $l$-2-th round of the encryption, and thus obtain a faulty ciphertext. By differential analysis, the value of the last subkey $K_{1}$ can be recovered. If the key size is 64 bits, then jump step (iv); else jump step (iii).

(iii) A random error is induced in the $l-6$-th round of the encryption, we could decrypt the right ciphertext by the subkey $K_{1}$ to obtain the input of the last four round, which is the output of the fifth round from the end. Repeat the same procedure to deduce the subkey $K_{2}$.

(iv) The secret key $K$ could be deduced on the basis of the key schedule.

\section{Single Byte Differential Fault Analysis on LED}

\subsection{Notations}

The following notations are used to describe the LED and its analysis.

Let $X \in\left(\{0,1\}^{4}\right)^{16}$ be the plaintext and $Y \in\left(\{0,1\}^{4}\right)^{16}$ be the ciphertext. Let $K_{1} \in\left(\{0,1\}^{4}\right)^{16}$ and $K_{2} \in\left(\{0,1\}^{4}\right)^{16}$ denote the subkeys from the secret key $K \in\left(\{0,1\}^{4}\right)^{16}$.

Let $A C_{j}^{d}, S C_{j}^{d}, S R_{j}^{d}$ and $M C_{j}^{d}$ represent the $j$-th 4bit output value of the AddConstants, SubCells, ShiftRows, and MixColumnsSerial layers in the $d$-th round with $1 \leq d \leq l$ and $0 \leq j \leq 15$, respectively.

Let $A R_{j}^{c}$ represents the $j$-th 4-bit output value of the AddRoundkey layer in the $c$-th 4 round with $1 \leq c \leq\lceil l / 4\rceil$ and $0 \leq j \leq 15$.

Let $\Delta A C_{j}^{d}, \Delta S C_{j}^{d}, \Delta S R_{j}^{d}$ and $\Delta M C_{j}^{d}$ represent the $j$ th 4-bit output difference of the AddConstants, SubCell, ShiftRows, and MixColumnsSerial layers in the $d$-th round with $1 \leq d \leq l$ and $0 \leq j \leq 15$, respectively.

Let $\Delta A R_{j}^{c}$ represents the $j$-th 4-bit output difference of the AddRoundkey layer in the $c$-th 4 round with $1 \leq c \leq\lceil l / 4\rceil$ and $0 \leq j \leq 15$.

The relationship between the input difference and output difference in the SubCells transformation is defined as follows:

$$
\begin{gathered}
I N\left(\Delta A C_{j}^{d}, \Delta S C_{j}^{d}\right)=\left\{A C_{j}^{d} \mid A C_{j}^{d} \in\{0,1\}^{4}, S\left(A C_{j}^{d}\right) \oplus\right. \\
\left.\left.S\left(A C_{j}^{d} \oplus \Delta A C_{j}^{d}\right)=\Delta S C_{j}^{d}\right\}, 0 \leq j \leq 15,1 \leq d \leq l\right\},
\end{gathered}
$$

where the $S$ represents a $4 \times 4$ substitution in the SubCells layer.

Let AddConstants ${ }^{-1}$, SubCells ${ }^{-1}$, ShiftRows ${ }^{-1}$, and MixColumnsSerial ${ }^{-1}$ represent the inverse operations of the AddConstants, SubCells, ShiftRows, and MixColumnsSerial transformations, respectively. 


\subsection{Attacking Procedure}

In this subsection, we apply the above basic idea and propose a novel differential fault analysis to recover the secret keys of LED-64 and LED-128. Our analysis is split into the following four successive steps:

(i) A ciphertext $Y$ is derived when an arbitrary plaintext $X$ is encrypted with a secret key $K$.

(ii) This step aims at recovering $K_{1}$ in the last round. The fault injection targets at the $l$-2-th round. As Fig. 2 shows, a fault may be induced on either $A C^{-2}$, $S C^{-2}$ or $S R^{1-2}$ whereas the approach is identical in either case. Note that any modification of one byte provokes the XOR-differences $\Delta M C^{-2}$ on $M C^{-2}$, $\triangle A C^{\mathrm{l}-1}$ on $A C^{\mathrm{l}-1}, \Delta S C^{\mathrm{l}-1}$ on $S C^{\mathrm{l}-1}, \Delta S R^{\mathrm{l}-1}$ on $S R^{\mathrm{l}-1}$, $\Delta M C^{\mathrm{l}-1}$ on $M C^{\mathrm{l}-1}, \triangle A C^{\mathrm{l}}$ on $A C^{\mathrm{l}}, \Delta S C^{\mathrm{l}}$ on $S C^{\mathrm{l}}$, $\Delta S R^{\mathrm{l}}$ on $S R^{\mathrm{l}}, \Delta M C^{\mathrm{l}}$ on $M C^{\mathrm{l}}$. These alter the original ciphertext $Y$ into the faulty ciphertext $Y^{*}$. We can observe that round could be represented by $\Delta S C_{j}^{l}$, where $0 \leq j \leq 15$. The transformation between inputs difference and outputs difference of the SubCells transformation is defined in the $l$-th round as below: $I N\left(\Delta A C_{j}^{l}, \Delta S C_{j}^{l}\right)=\left\{A C_{j}^{l} \mid A C_{j}^{l} \in\{0,1\}^{4}\right.$,

$$
\left.S\left(A C_{j}^{l}\right) \oplus S\left(A C_{j}^{l} \oplus \Delta A C_{j}^{l}\right)=\Delta S C_{j}^{l}, 0 \leq j \leq 15\right\} .
$$

The above equation, in conjunction with a pair of right and faulty ciphertexts, allow to infer a relation between $\triangle A C^{l}$ and $\Delta S C^{l}$. It is helpful to restrict a list of possible candidates for the value of $K_{1}$. The MixColumnsSerial layer propagates one single byte fault to four-byte differences in the input of the SubCells transformation. If we do brute force search for the input of the SubCells transformation, the complexity to recover one subkey is up to $2^{64}$. This kind of search is not really practical.

We propose an effective approach to select the input difference of the SubCells transformation, so the input of the SubCells transformation could be obtained with less complexity. We take the derivation of $A C^{l}$ as an example. We observe that

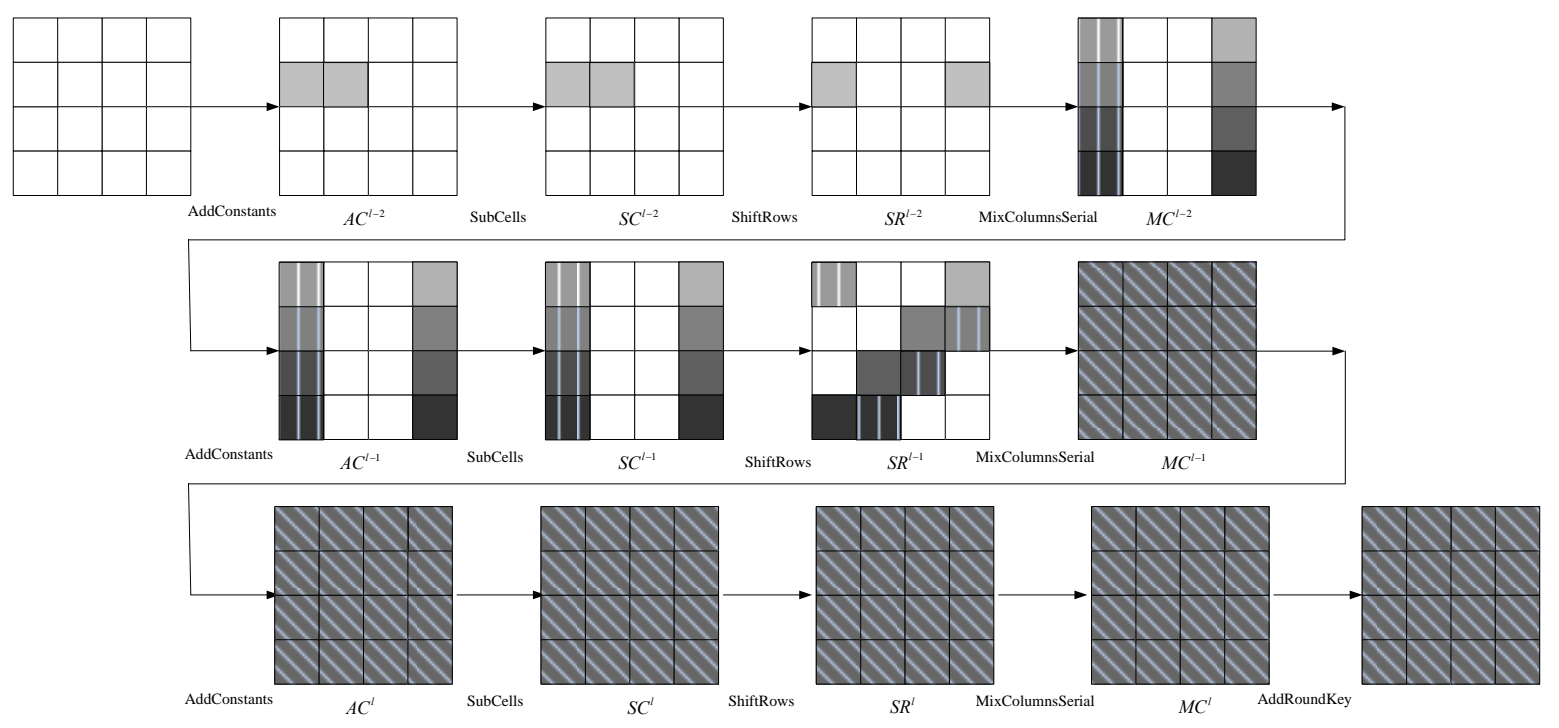

Fig. 2 One single-byte fault progagation path in the last three rounds of the LED cipher

$$
\begin{aligned}
\Delta Y & =Y \oplus Y^{*} \\
\Delta M C^{l} & =\left(Y \oplus K_{1}\right) \oplus\left(Y^{*} \oplus K_{1}\right)=\Delta Y \\
\Delta S C^{l} & =\operatorname{ShiftRows}^{-1}\left(\Delta S R^{l}\right) \\
& =\text { ShiftRows }^{-1}\left(\text { MixColumnsSerial }^{-1}\left(\Delta M C^{l}\right)\right) \\
& =\text { ShiftRows }^{-1}\left(\text { MixColumnsSerial }^{-1}(\Delta Y)\right)
\end{aligned}
$$

The output difference of the $j$-th S-box in the $l$-th one single byte error can lead to two 4-byte differences independently after the computation of the diffusion layer in $A C^{l-1}$, and the differences could result in the 64-bit differences in $\triangle A C^{l}$ as Fig. 2 shows. This important property helps to do brute force search on $\triangle A C^{l}$. We separate the one-byte fault into two 4-bit values. This approach simplifies the analysis and improve the attacking efficiency. 
On the basis of one single-byte inducing fault, there are four types of the values of $\triangle A C^{l}$ in Fig. 3. In every type value, the set of $\Delta A C^{l}$ could be separated into two subsets of $\triangle A C^{l}$ which are easier to compute. On the basis of two subsets, the values of $\triangle A C^{l}$ shows the linear relationships between different columns.

The equations are derived as follows:

$$
\begin{aligned}
& \left(\Delta Y_{0}, \Delta Y_{7}, \Delta Y_{10}, \Delta Y_{13}\right)= \\
& \left(S\left(A C_{0}^{l}\right) \oplus S\left(A C_{0}^{l} \oplus \Delta A C_{0}^{l}\right), S\left(A C_{4}^{l}\right) \oplus S\left(A C_{4}^{l} \oplus \Delta A C_{4}^{l}\right), \quad\right. \text { (8) } \\
& \left.S\left(A C_{8}^{l}\right) \oplus S\left(A C_{8}^{l} \oplus \Delta A C_{8}^{l}\right), S\left(A C_{12}^{l}\right) \oplus S\left(A C_{12}^{l} \oplus \Delta A C_{12}^{l}\right)\right), \\
& \left(\Delta Y_{1}, \Delta Y_{4}, \Delta Y_{11}, \Delta Y_{14}\right)= \\
& \left(S\left(A C_{1}^{l}\right) \oplus S\left(A C_{1}^{l} \oplus \Delta A C_{1}^{l}\right), S\left(A C_{5}^{l}\right) \oplus S\left(A C_{5}^{l} \oplus \Delta A C_{5}^{l}\right),(9)\right. \\
& \left.S\left(A C_{9}^{l}\right) \oplus S\left(A C_{9}^{l} \oplus \Delta A C_{9}^{l}\right), S\left(A C_{13}^{l}\right) \oplus S\left(A C_{13}^{l} \oplus \Delta A C_{13}^{l}\right)\right),
\end{aligned}
$$
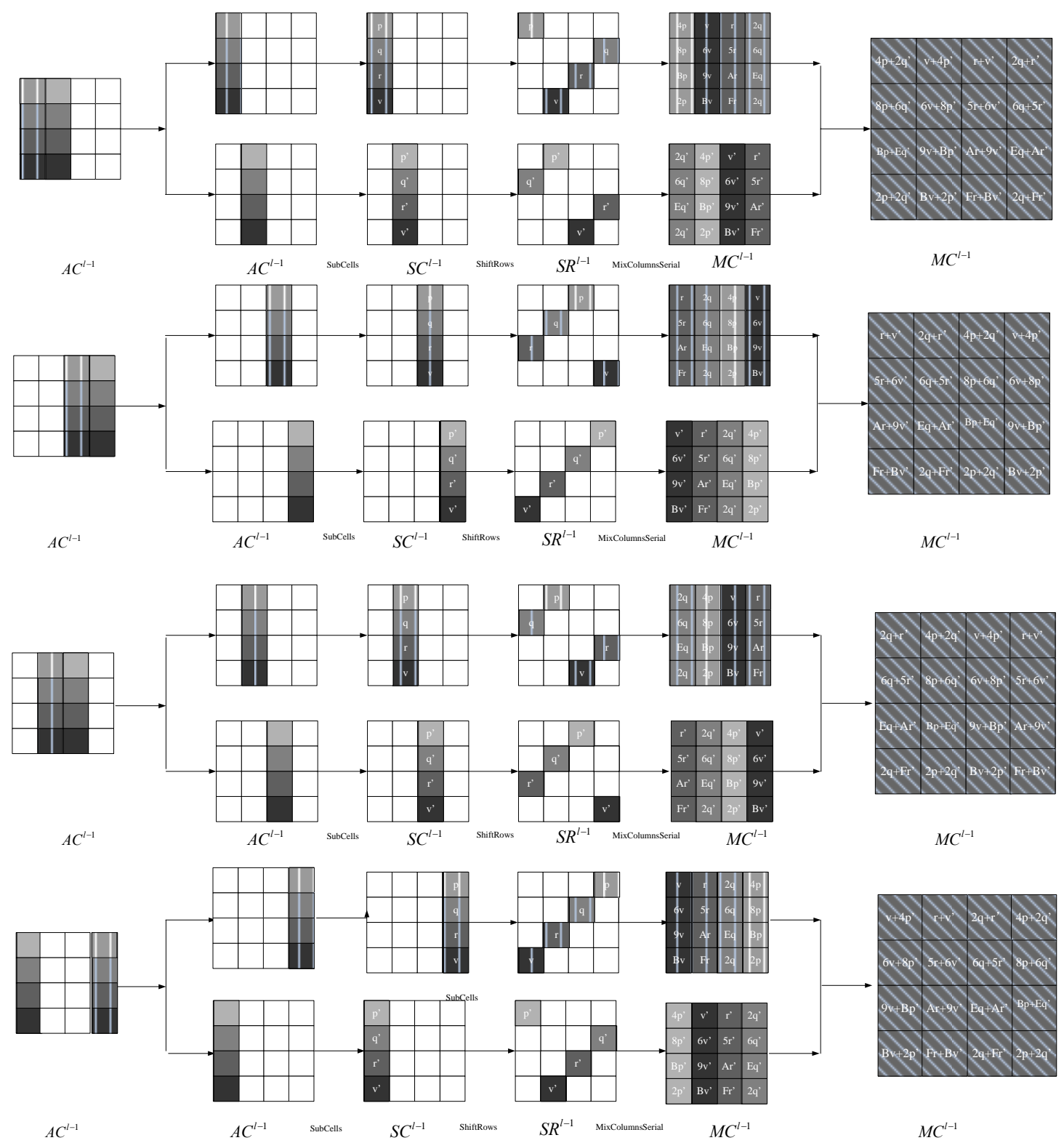

Fig. 3 The four relationships among the fault progagation values in the LED cipher 


$$
\begin{aligned}
& \left(\Delta Y_{2}, \Delta Y_{5}, \Delta Y_{8}, \Delta Y_{15}\right)= \\
& \left(S\left(A C_{2}^{l}\right) \oplus S\left(A C_{2}^{l} \oplus \Delta A C_{2}^{l}\right), S\left(A C_{6}^{l}\right) \oplus S\left(A C_{6}^{l} \oplus \Delta A C_{6}^{l}\right), \quad\right. \text { (10) } \\
& \left.\quad S\left(A C_{10}^{l}\right) \oplus S\left(A C_{10}^{l} \oplus \Delta A C_{10}^{l}\right), S\left(A C_{14}^{l}\right) \oplus S\left(A C_{14}^{l} \oplus \Delta A C_{14}^{l}\right)\right), \\
& \left(\Delta Y_{3}, \Delta Y_{6}, \Delta Y_{9}, \Delta Y_{12}\right)= \\
& \left(S\left(A C_{3}^{l}\right) \oplus S\left(A C_{3}^{l} \oplus \Delta A C_{3}^{l}\right), S\left(A C_{7}^{l}\right) \oplus S\left(A C_{7}^{l} \oplus \Delta A C_{7}^{l}\right), \quad(11)\right. \\
& \left.S\left(A C_{11}^{l}\right) \oplus S\left(A C_{11}^{l} \oplus \Delta A C_{11}^{l}\right), S\left(A C_{15}^{l}\right) \oplus S\left(A C_{15}^{l} \oplus \Delta A C_{15}^{l}\right)\right) .
\end{aligned}
$$

When the random fault influences the 1st, 2nd, 3rd or 4th columns of $A C^{l-1}$, the value of $\triangle A C^{l}$ could be represented by

$$
\begin{aligned}
& \Delta A C^{l}=\left(\begin{array}{cccc}
\Delta A C_{0}^{l} & \Delta A C_{1}^{l} & \Delta A C_{2}^{l} & \Delta A C_{3}^{l} \\
\Delta A C_{4}^{l} & \Delta A C_{5}^{l} & \Delta A C_{6}^{l} & \Delta A C_{7}^{l} \\
\Delta A C_{8}^{l} & \Delta A C_{9}^{l} & \Delta A C_{10}^{l} & \Delta A C_{11}^{l} \\
\Delta A C_{12}^{l} & \Delta A C_{13}^{l} & \Delta A C_{14}^{l} & \Delta A C_{15}^{l}
\end{array}\right) \in \\
& \left\{\begin{array}{cccc}
4 p+2 q^{\prime} & v+4 p^{\prime} & r+v^{\prime} & 2 q+r^{\prime} \\
8 p+6 q^{\prime} & 6 v+8 p^{\prime} & 5 r+6 v^{\prime} & 6 q+5 r^{\prime} \\
B p+E q^{\prime} & 9 v+B p^{\prime} & A r+9 v^{\prime} & E q+A r^{\prime} \\
2 p+2 q^{\prime} & B v+2 p^{\prime} & F r+B v^{\prime} & 2 q+F r^{\prime}
\end{array}\right), \\
& \left(\begin{array}{cccc}
r+v^{\prime} & 2 q+v^{\prime} & 4 p+2 q^{\prime} & v+4 p^{\prime} \\
5 r+6 v^{\prime} & 6 q+5 r^{\prime} & 8 p+6 q^{\prime} & 6 v+8 p^{\prime} \\
A r+9 v^{\prime} & E q+A r^{\prime} & B p+E q^{\prime} & 9 v+B p^{\prime} \\
F r+B v^{\prime} & 2 q+F r^{\prime} & 2 p+2 q^{\prime} & B v+2 p^{\prime}
\end{array}\right), \\
& \left(\begin{array}{cccc}
2 q+r^{\prime} & 4 p+2 q^{\prime} & v+4 p^{\prime} & r+v^{\prime} \\
6 q+5 r^{\prime} & 8 p+6 q^{\prime} & 6 v+8 p^{\prime} & 5 r+6 v^{\prime} \\
E q+A r^{\prime} & B p+E q^{\prime} & 9 v+B p^{\prime} & A r+9 v^{\prime} \\
2 q+F r^{\prime} & 2 p+2 q^{\prime} & B v+2 p^{\prime} & F r+B v^{\prime}
\end{array}\right), \\
& \left(\begin{array}{cccc}
v+4 p^{\prime} & r+v^{\prime} & 2 q+r^{\prime} & 4 p+2 q^{\prime} \\
6 v+8 p^{\prime} & 5 r+6 v^{\prime} & 6 q+5 r^{\prime} & 8 p+6 q^{\prime} \\
9 v+B p^{\prime} & A r+9 v^{\prime} & E q+A r^{\prime} & B p+E q^{\prime} \\
B v+2 p^{\prime} & F r+B v^{\prime} & 2 q+F r^{\prime} & 2 p+2 q^{\prime}
\end{array}\right), \\
& \left.p, p^{\prime}, q, q^{\prime}, r, r^{\prime}, v, v^{\prime} \in\{0,1\}^{4}\right\} \text {. }
\end{aligned}
$$

Thus we could derive all possible values of $A C^{l}$. By inducing random faults repeating the above approach and until $A C^{l}$ has only one value. All bytes of $K_{1}$ could be deduced as follows:

$K_{1}=Y \oplus M C^{l}$

$=Y \oplus \operatorname{MixColumnsSerial}\left(S R^{l}\right)$

$=Y \oplus \operatorname{Mix}$ ColumnsSerial $\left(\operatorname{ShiftRows}\left(\operatorname{SubCells}\left(A C^{l}\right)\right)\right)$. (iii) After recovering the subkey $K_{1}$, we could perform the following procedure for $K_{2}$ of LED-128. We make advantage of the previous step (ii) to derive the output of the $l-4$-th rounds, and induce faults into the $l-6$-th round. After computing the input difference $\Delta A C^{l-4}$ and output difference $\Delta S C^{l-4}$ in the S-boxes transformation, we decrease the number of $A C^{l-4}$ candidates by repeating the proposed method and the collected faulty ciphertexts, until the set of $A C^{l-4}$ candidates has only one element. All bytes of $A C^{l-4}$ could be deduced. Thus, all bytes of $K_{2}$ could be deduced as follows:

$$
\begin{aligned}
& K_{2}=M C^{l-4} \oplus A R^{12} \\
& =\operatorname{MixColumnsSerial}\left(\operatorname{ShiftRows}\left(\operatorname{SubCells}\left(A C^{l-4}\right)\right)\right) \oplus \\
& \text { AddConstants(SubCells(ShiftRows(MixColumnsSerial( } \\
& \text { AddConstants(SubCells(ShiftRows(MixColumnsSerial( (14) } \\
& \text { AddConstants(SubCells(ShiftRows(MixColumnsSerial( } \\
& \text { AddConstants(SubCells(ShiftRows(MixColumnsSerial( } \\
& \left.\left.\left.\left.\left.\left.\left.\left.\left.\left.\left.\left.\left.\left.Y \oplus K_{1}()\right)\right)\right)\right)\right)\right)\right)\right)\right)\right)\right)\right)\right)\right) \text { ). }
\end{aligned}
$$

(iv) On the basis of the key schedule, $K=K_{1}$ in LED-64, and $K=K_{1} \| K_{2}$ in LED-128.

\section{Attacking Complexity}

We summarize the attacking procedure to select subkey candidates for the 64-bit and 128-bit secret keys. The time complexity of brute-force search for one fault injection is

$$
u=2^{x \cdot s} \cdot\left(2^{s} \cdot\left\lceil\frac{n}{s}\right\rceil\right),
$$

where $n$ denotes the size of the SubCells transformation, $s$ denotes the input size of one S-box, and $x$ denotes the number of S-boxes in parallel.

In addition, an estimation of the number of faults necessary for the attack to be successful is vital. In the attacking procedure, the number of faulty ciphertexts to recover a subkey depends on the fault location and the fault model.

We take the derivation of $K_{1}$ as an example. On the property of the SubCells layer, if $K_{1}$ is a subkey candidate, then $K_{1} \oplus \Delta M C^{l}$ may be another subkey candidate. In other words, if the input candidates set of S-boxes is not null, then the input $A C^{l}$ may have several candidates. Thus, there are some candidates of $M C^{l}$. It indicates that $K_{1}$ may have some possible elements.

In the single-byte fault model, a random error could be induced at any round of the encryption. If the fault occurs in the last round, then only one single byte in the 
input of the SubCells transformation will change, which could recover at most one byte of the last subkey by DFA. To recover the last subkey, it is necessary to induce many errors into different bytes.

If the fault is induced at an ideal location before the last round, then the inputs difference and outputs difference of the SubCells transformation in this round contain only one nonzero byte. However, the output difference of ShiftRows and MixColumnsSerial has multibytes owing to the diffusion of linear transformation. Thus, the input difference of MixColumnsSerial in the last round contains multibytes after the computation of the last several rounds. The above idea is applied in the attacking procedure to improve the efficiency of fault injection.

Since at least two errors can make one element in the intersection of $K_{1}$, we continue deriving intersection of subkey candidates sets until the intersection has only one element. Thus, at least two fault ciphertexts are required to derive multibytes of one subkey. The theoretical minimum number of faulty ciphertexts to recover one subkey is defined as

$$
w=\left\{\begin{array}{cl}
0 & \text { if } \mathrm{m}=0, \\
\left\lceil\frac{2 n}{m}\right\rceil & \text { if } 1 \leq \mathrm{m} \leq \mathrm{n},
\end{array}\right.
$$

where $n$ represents the size of the SubCells layer, and $m$ represents the maximum number of bits in a subkey derived by two faulty ciphertexts. To derive the subkey, the value of $m$ equals the number of bits in the nonzero output difference of the nonlinear transformation in this round. If $m=0$, then there is no bits of a subkey derived and thus $w=0$.

Thus, the overall attacking complexity to recover a secret key is

$$
u \cdot w \cdot g= \begin{cases}0 & \text { if } \quad m=0, \\ 2^{(x+1) \cdot s+1} \cdot\left\lceil\frac{n^{2} \cdot g}{s \cdot m}\right\rceil & \text { if } 1 \leq m \leq n,\end{cases}
$$

where $g$ denotes the number of subkeys to recover a secret key, $n$ denotes the size of the SubCells layer, $s$ denotes the input size of one S-box, $x$ denotes the number of S-boxes in parallel and $m$ represents the maximum number of bits in a subkey derived by two faulty ciphertexts.

For a 64-bit secret key, the attacking complexity in theory is about

$$
2^{25}\left(=2^{(4+1) \cdot 4+1} \cdot\left\lceil\frac{64^{2} \cdot 1}{4 \cdot 64}\right\rceil\right)
$$

where $g=1, n=64, x=4, s=4, m=64$, and $v=2$. For a 128 bit secret key, the attacking complexity in theory is about

$$
2^{26}\left(=2^{(4+1) \cdot 4+1} \cdot\left\lceil\frac{64^{2} \cdot 2}{4 \cdot 64}\right\rceil\right)
$$

for $g=2, n=64, x=4, s=4, m=64$, and $v=2$.

\section{Experimental Results}

We implemented our attack on a PC using Visual C++ 8.0 Compiler on a $2.53 \mathrm{GHz}$ celeron with 2GB memory. The fault induction was simulated by computer software. In this situation, we ran the attack algorithm to 1000 encryption unit with a random generated key.

Fig. 4 shows the number of subkey candidates in three intersections of subkey candidates to recover one subkey. We define accuracy, reliability and latency for evaluating the experimental results in detail.

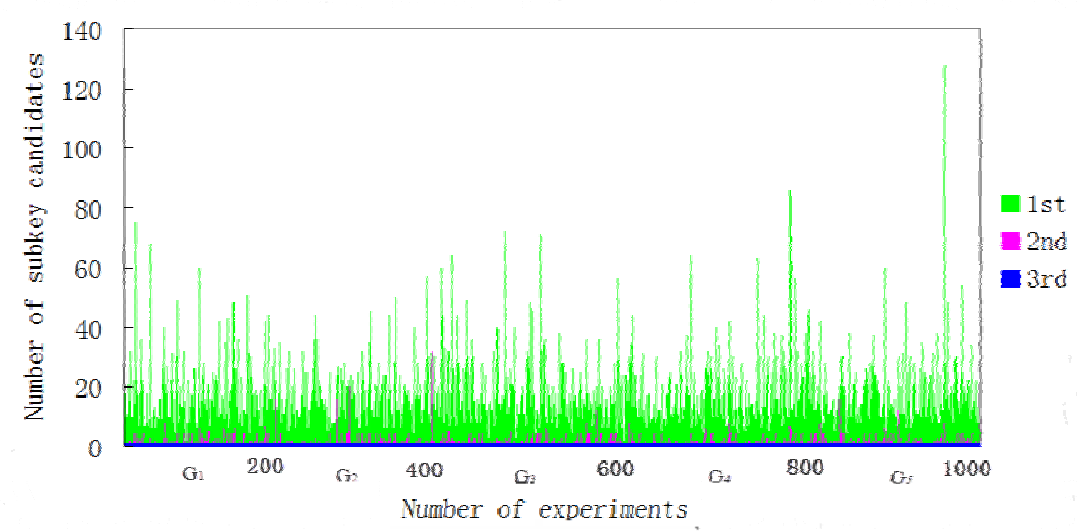

Fig. 4 (color) Three intersections of subkey candidates in the 1000 experiments 
Accuracy is a measure that defines how close the number of subkey candidates are to the true number of subkey candidates. Basically, the closer the experimental number of subkey candidates is to the true number, the more accurate the experiment is. Thus, we consider the Root Mean-Square Error(RMSE) to measure the accuracy, where RMSE is given by

$$
R M S E=\sqrt{\frac{1}{N} \sum_{e=1}^{N}\left[h_{\text {measured }}(e)-h_{\text {true }}\right]},
$$

where $N$ is the number of experiments in a set and $e$ is the index of the experiment, $h_{\text {measured }}$ is the number of subkey candidates, and $h_{\text {true }}$ is the number of ture subkeys. As we know, there is only one true subkey. The closer the RMSE value is to 0 , the more accurate the experiments are. We divide 1000 experiments as 5 groups in average, denoted as $G_{1}, G_{2}, G_{3}, G_{4}$ and $G_{5}$. The RMSE values for every intersections of subkey candidates are shown in Table 1 , where $N=200, h_{\text {true }}=1$ and $e \in\{1, \cdots, 1000\}$. Thus, the 3rd intersection of subkey candidates is completely accurate, and we could derive the subkey in this intersection. That is, 4 fault ciphertexts are required to recover one subkey. Furthermore, the accuracy in every group for the same interaction is similar or equal.

Table 1. One subkey recovery on accuracy by RMSE

\begin{tabular}{cccc}
\hline Groups & $\begin{array}{c}\text { 1st } \\
\text { intersection }\end{array}$ & $\begin{array}{c}\text { 2nd } \\
\text { intersection }\end{array}$ & $\begin{array}{c}\text { 3rd } \\
\text { intersection }\end{array}$ \\
\hline $\mathrm{G}_{1}$ & 19.46 & 1.79 & 0 \\
$\mathrm{G}_{2}$ & 19.54 & 2.99 & 0 \\
$\mathrm{G}_{3}$ & 19.38 & 1.62 & 0 \\
$\mathrm{G}_{4}$ & 19.67 & 1.24 & 0 \\
$\mathrm{G}_{5}$ & 20.05 & 1.89 & 0 \\
\hline
\end{tabular}

Table 2. One subkey recovery on reliability

\begin{tabular}{cccc}
\hline Groups & $\begin{array}{c}\text { 1st } \\
\text { intersection }\end{array}$ & $\begin{array}{c}\text { 2nd } \\
\text { intersection }\end{array}$ & $\begin{array}{c}\text { 3rd } \\
\text { intersection }\end{array}$ \\
\hline $\mathrm{G}_{1}$ & 0 & $65.0 \%$ & $100 \%$ \\
$\mathrm{G}_{2}$ & 0 & $64.0 \%$ & $100 \%$ \\
$\mathrm{G}_{3}$ & 0 & $66.0 \%$ & $100 \%$ \\
$\mathrm{G}_{4}$ & 0 & $67.5 \%$ & $100 \%$ \\
$\mathrm{G}_{5}$ & 0 & $65.5 \%$ & $100 \%$ \\
\hline
\end{tabular}

Reliability is the ratio of successful experiments out of all experiments made. If the attacker could derive only one subkey, the experiment is successful. Referring to Table 2, it is observed that the ratio of successful experiments in the 1st, 2nd and 3rd intersections of subkey candidates are $0,65.6 \%$ and
$100 \%$, respectively. That is, the reliability is $100 \%$ if the attacker induces 4 random faults to break a subkey. Furthermore, the reliability in every group for the same interaction is similar or equal.

Latency is the time from the first fault injection to the recovery of the subkey in our software simulation. It is measured in seconds. Fig. 5 shows that the latency of 1000 experiments. The time of $74.5 \%$ experiments is between $0.1 \mathrm{~s}$ and $0.2 \mathrm{~s}$.

Thus, 4 faulty ciphertexts are required to recover one subkey. The proposed DFA method requires 4 faulty ciphertexts to recover the 64-bit secret key and 8 faulty ciphertexts to recover 128-bit secret keys.

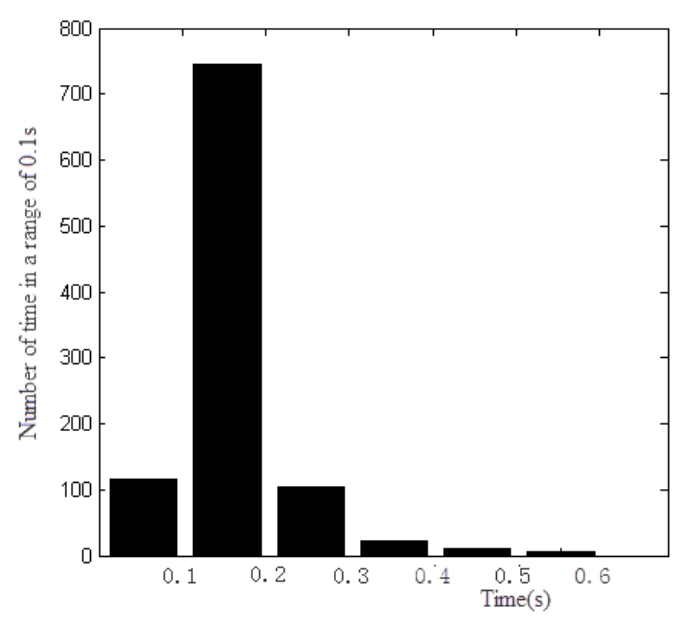

Fig. 5 One subkey recovery on latency

On the basis of the number of faulty ciphertexts in our simulated experiments, the attacking complexity in practice is

$$
2^{26}\left(=2^{(4+1) \cdot 4} \cdot\left\lceil\frac{64^{2} \cdot 1}{4 \cdot 64}\right\rceil \cdot 4\right)
$$

to recover one subkey. Thus, the attacking complexity is about

$$
2^{26}\left(=2^{(4+1) \cdot 4} \cdot\left\lceil\frac{64^{2} \cdot 1}{4 \cdot 64}\right\rceil \cdot 4\right)
$$

and

$$
2^{27}\left(=2^{(4+1) \cdot 4} \cdot\left\lceil\frac{64^{2} \cdot 1}{4 \cdot 64}\right\rceil \cdot 8\right)
$$

to break the LED-64 and LED-128 by the single byte differential fault analysis, respectively.

\section{Conclusion}

This paper examines single byte differential fault analysis on LED in software implementation. It shows that LED is vulnerable to the single byte differential 
fault analysis. In the byte-oriented fault model, only 4 or 8 ciphertexts in average is required to obtain the 64-bit and 128-bit secret key of LED, respectively. Our work provides a new reference to fault analysis on other block ciphers.

In consequence, we are working on fault analysis on LED in hardware implementation. Furthermore, future analysis should be able to support more fault locations of LED, such as the key schedule.

\section{Acknowledgements}

The authors are grateful to the editors and the anonymous reviewers for their helpful comments. This work is supported by the National Natural Science Foundation of China under Grant No. 61003278 and No. 61073150, the Opening Project of Shanghai Key Laboratory of Integrate Administration Technologies for Information Security, the open research fund of State Key Laboratory of Information Security and the Fundamental Research Funds for the Central Universities.

\section{References}

1. J. Guo and T. Peyrin, A. Poschmann, et al. The LED block cipher, in 13th Int. W orkshop Cryptographic Hardware and Embedded Systems, eds. B. Preneel and T. Takagi (Nara, Japan, 2011), pp. 326-341.

2. E. Biham and A. Shamir. Differential fault analysis of secret key cryptosystems, in 17th Annual Int. Cryptology Conf., eds. S. Burton and Jr. Kaliski (California, USA, 1997) , pp. 513-525.

3. J. Blomer and J. P. Seifert. Fault based cryptanalysis of theadvanced encryption standard (AES), in 7th Int. Conf. Financial Cryptography, eds. R. N. Wright (Guadeloupe, French West Indies, 2003), pp. 162-181.

4. C. N. Chen and S. M. Yen. Differential fault analysis on AES key schedule and some countermeasures. in Proc. Australasian Conf. Information Security and Privacy, eds. H. Wang, J. Pieprzyk and V. Varadharajan (Wollongong, Australia, 2004), pp. 118-129.

5. P. Dusart, G. Letourneux and O. Vivolo. Differential fault analysis on AES. in 1st Int. Conf. Applied Cryptography and Network Security, eds. J. Zhou, M. Yung and Y. Han (Kunming, China, 2003), pp. 293-306.

6. C. Giraud. DFA on AES. in 4th Int. Conf. Advanced Encryption Standard, eds. H. Dobbertin, V. Rijmen and A. Sowa (Bonn, Germany, 2004), pp. 27-41.

7. P. Gilles and J. J. Quisquater. A differential fault attack technique against SPN tsructures, with application to the AES and KHAZAD, in 5th Int. Workshop Cryptographic Hardware and Embedded Systems, eds. C. D. Walter, C. L. Koc and C. Paar (Cologne, Germany, 2003), pp. 77-88.

8. M. Amir, T. M. S. Mohammad and S. Mahmoud. A generalized method of differential fault attack against
AES cryptosystem. in 8th Int. Workshop Cryptographic Hardware and Embedded $S$ ystems, eds. L. Goubin and M. Matsui (Yokohama, Japan, 2006), pp. 91-100.

9. L. Hemme. A differential fault analysis against early rounds of (Triple-) DES, in 6th Int. Workshop Cryptographic Hardware and Embedded Systems, eds. M. Joye and J. J. Quisquater (Cambridge, MA, USA, 2004), pp. 254-267.

10. E. Biham, L. Granboulan and P. Q. Nguyon. Impossible fault analysis of RC4 and differential fault analysis of RC4, in 12th Int. Workshop Fast Software Encryption, eds. H. Gilbert and H. Handschuh (Paris, France, 2005), pp. 359-367.

11. W. Li, D. Gu, J. Li, Z. Liu and Y. Liu. Differential fault analysis on Camellia. J. Syst. Software 83(2010) pp. 844851.

12. W. Li, D. Gu and J. Li. Differential fault analysis on the ARIA algorithm, Inform. Sciences 178(19)(2008) 3727 3737.

13. L. Zhang and W. Wu, Differential fault analysis on SMS4. Chinese J. Comput. 29(9)(2008) 1596-1602.

14. R. Li, B. Sun, C. Li and J. You. Differential fault analysis on SMS4 using a single fault. Inform. Process. Lett. 111(4) (2011)156-163.

15. J. Li and D. Gu. Differential fault attack on PRESENT block cipher, in Proc. Annual Conf. Chinese Association for Cryptologic Research, eds. D. Pei and B. Yang (Guangzhou, China, 2009), pp. 3-13. 